Published in final edited form as:

Ann Epidemiol. 2009 June ; 19(6): 388-395. doi:10.1016/j.annepidem.2008.12.018.

\title{
Occupational Exposure to Metribuzin and the Incidence of Cancer in the Agricultural Health Study
}

\author{
John Oliver L. DeLancey, MPH ${ }^{1,2}$, Michael C. R. Alavanja, Dr.P.H. ${ }^{3}$, Joseph Coble, Sc. D $^{3}$, \\ Aaron Blair, Ph.D ${ }^{3}$, Jane A. Hoppin, Sc. ${ }^{4}$, Harland D. Austin, Sc.D ${ }^{1}$, and Laura E. Beane \\ Freeman, Ph. $\mathbf{D}^{3}$ \\ 1 Emory University Rollins School of Public Health, Department of Epidemiology \\ 2American Cancer Society \\ 3Division of Cancer Epidemiology and Genetics, National Cancer Institute \\ 4Epidemiology Branch, National Institute of Environmental Health Sciences
}

\begin{abstract}
Purpose-Little is known about the potential carcinogenicity of the triazinone herbicide metribuzin. We evaluated the association between metribuzin use and cancer risk in the Agricultural Health Study, a prospective cohort study of licensed pesticide applicators in Iowa and North Carolina.

Methods-Applicators ( $\mathrm{n}=23,072)$ provided information on metribuzin use on a self-administered questionnaire at enrollment (1993-1997). Among metribuzin users $(n=8,504)$, there were 554 incident cancer cases. We used multivariable Poisson regression to evaluate potential associations between metribuzin use and cancer incidence using two quantitative exposure metrics, lifetime days and intensity-weighted lifetime days.
\end{abstract}

Results-Using intensity-weighted lifetime days, the rate ratio (RR) and 95\% confidence interval (CI) for the highest exposed tertile for lymphohematopoietic malignancies was 2.09 (95\% CI: $0.99-4.29$ ), p-trend $=0.02$ and 2.42 (95\% CI: 0.82, 7.19), p-trend=0.08 for leukemia. For NonHodgkin lymphoma, the RR was 2.64 (95\% CI: 0.76, 9.11), p-trend=0.13 for lifetime days and 2.52 ( $95 \%$ CI: $0.66-9.59$ ), p-trend $=0.13$ for intensity-weighted lifetime days. Patterns of association were similar for both exposure metrics, but associations were generally weaker than for intensity-weighted days.

Conclusions-The results from this study suggest a potential association between metribuzin use and certain lymphohematopoietic malignancies; however, having not been observed previously caution should be used in interpretation.

\section{Keywords}

pesticides; cancer; occupation; metribuzin; Agricultural Health Study

\section{INTRODUCTION}

Metribuzin is a selective triazinone herbicide that is used to control broadleaf weeds and grasses in vegetable and field crops, turf grasses, and non-crop areas by preventing electron transport for photosynthesis (1). U.S. average annual use for the years 1990-1994 is estimated at 2.8 million pounds per year of active ingredient, treating approximately 8.5 million acres (2). Metribuzin is considered "moderately hazardous" by the World Health Organization (WHO) (3), "slightly toxic" (toxicity class III) through oral and inhalation routes of exposure, and "practically non-toxic" by the dermal route of exposure (toxicity class IV) by the United States 
Environmental Protection Agency (US EPA) (1). Metribuzin has been classified by the EPA as a Group D chemical, "not classifiable as to human carcinogenicity" due to inadequate carcinogenicity data from animal bioassays and the lack of carcinogenicity data in humans (4).

Tests for the mutagenicity and genotoxicity of metribuzin have produced inconsistent results. Negative results were observed in an in vivo genotoxicity study using the somatic mutation and recombination test in Drosophila melanogaster wings (5). Statistically significant increases in sister chromatid exchanges (SCE) were observed in human lymphocytes in vitro when treated with metribuzin and a metabolic mixture, but not when treated with metribuzin alone, although cytotoxic effects expressed as cellular death were observed (6). Genotoxicity studies in vitro produced negative results using a bacterial reversion assay in E. coli (7) and a microtitration SOS microplate assay in E. coli with and without metabolic activation (8), but a similar study used a modified SOS microplate assay in E. coli that yielded mild positive genotoxic effects of metribuzin (9). A study in Rana catesbeiana tadpoles found significant DNA damage at all doses of metribuzin exposure, but no dose-response effect using the alkaline single-cell gel DNA electrophoresis (comet) assay (10). A genotoxic study in vitro using ${ }^{32} \mathrm{P}-$ postlabeling with both nuclease P1 enrichment and butanol enrichment showed that metribuzin was positive for DNA adduct formation (11). Bleeke et al. reported that 90 percent of administered metribuzin was excreted within 5 days, with roughly equal amounts excreted in urine and feces (12).

Two-year feeding studies conducted for regulatory evaluation showed that metribuzin treatment (up to $480 \mathrm{mg} / \mathrm{kg} / \mathrm{day}$ ) did not result in an increase of tumor incidence in mice and rats (2). Metribuzin did, however, yield high mortality, decreased body weight, and pathologic changes in the liver, kidneys, uterus, and mammary glands in dogs exposed to metribuzin at a rate of $55.7 \mathrm{mg} / \mathrm{kg} / \mathrm{day}(2)$.

To our knowledge, there are only two epidemiologic studies examining an association between metribuzin exposure and cancer. The first examined the association between metribuzin use and the incidence of Non-Hodgkin's lymphoma (NHL). It was conducted as part of a pooled analysis of data from three case-control studies of NHL from the 1980s to examine ever/never pesticide use in farmers as risk factors for NHL (13). In this study, ever use of metribuzin was not associated with increased incidence of NHL. The second was a case-control study of pesticide use and risk of glioma, conducted as part of the Nebraska Health Study II. This study did find a significantly increased risk of glioma for the 9 exposed cases (14).

Due to the dearth of epidemiologic data regarding whether an association exists between metribuzin exposure and cancer (4), the aim of our research was to examine the association between metribuzin use and the risk of cancer at select cancer sites among pesticide applicators enrolled in the Agricultural Health Study using data collected at enrollment (15).

\section{MATERIALS AND METHODS \\ Cohort enrollment and follow-up}

The Agricultural Health Study (AHS) is a prospective cohort of 57,310 licensed pesticide applicators and 32,347 of their spouses in Iowa and North Carolina (15). The AHS cohort has previously been described in detail (15). Briefly, the cohort includes 52,395 private applicators (mostly farmers) from Iowa and North Carolina and 4,916 commercial applicators from Iowa who applied pesticides for a pest control company or other business. The full cohort comprised $82 \%$ of pesticide applicators who applied for licenses to apply restricted-use pesticides during the enrollment period (December 13, 1993 to December 31, 1997) from Iowa and North Carolina. Participants were linked to population-based cancer registries in Iowa and North 
Carolina by full name, sex, date of birth, and Social Security Number to ascertain cancer incidence and to State death registries and the National Death Index to determine vital status on a yearly basis. Incident cancer cases that occurred between enrollment and December 31, 2004 were coded in accordance with the International Classification of Diseases for Oncology (ICD-O 2).

Participants completed a self-administered questionnaire at enrollment to gather information on demographic variables (including smoking history, alcohol consumption, education, family history of cancer), detailed exposure information on 22 pesticides, ever/never exposure information on 28 additional pesticides, pesticide application methods, use of personal protective equipment, pesticide mixing, and equipment handling and repair. All participants were also given a take-home questionnaire that asked more detailed information on pesticides for which the enrollment questionnaire provided only ever/never use (including metribuzin). Both questionnaires are available at the Agricultural Health Study website: www.aghealth.org/questionnaires.html.

Of those participants who completed the enrollment questionnaire, 47 percent also completed the take-home questionnaire. The characteristics of those who completed the take-home questionnaire were similar to those who only completed the enrollment questionnaire; the most notable difference being that participants who completed the take-home questionnaire were slightly older than those who only completed the enrollment questionnaire, which may result in higher cumulative lifetime days of exposure (16).

Participants with a cancer diagnosis prior to enrollment were excluded from the analysis $(n=1,084)$. Detailed information on metribuzin use was determined from the take-home questionnaire, so participants who did not complete the take-home questionnaire $(n=31,559)$ were also excluded. Females $(n=667)$ were also excluded because only 68 women reported using metribuzin on the take-home questionnaire. After these exclusions, a total of 23,072 cohort members who provided detailed metribuzin use information were included in analyses.

\section{Exposure Assessment}

Two quantitative exposure estimates were calculated from responses to the take-home questionnaire: 1) total days of lifetime use and 2) intensity-weighted days of lifetime use. The total lifetime days of metribuzin use variable was calculated by multiplying the midpoints of categorical variables for number of years of exposure and average number of days per year of exposure. For example, a subject who answered that they applied metribuzin for 6-10 years at an average of 10-19 days per year was estimated to have received 116 lifetime days of exposure ( 8 years $\times 14.5$ days/year). The intensity-weighted days of lifetime exposure variable was calculated by multiplying the total days of lifetime exposure variable by an intensity factor estimated using an algorithm that incorporates information from the enrollment questionnaire and published monitoring data. The intensity-weighting algorithm accounts for various factors which may modify exposure and is calculated as follows: Intensity level $=[($ mixing status + application method + equipment repair status) $\times$ personal protective equipment use] (17).

Both lifetime exposure days and intensity-weighted exposure days were categorized into ever/ never categories and tertiles of exposure (low, medium, and high) based on the distribution of exposure in cancer cases.

\section{Statistical Analysis}

Poisson regression analyses were performed using Stata statistical software program (Release 9.0, Stata Corporation, College Station, TX; 2005) to calculate rate ratios (RR) and 95 percent confidence intervals (CI) for the association between metribuzin exposure and the incidence 
of cancer. Results are reported for all cancer sites and cancer sites with at least five cases in each exposure category for total lifetime days of exposure and intensity-weighted lifetime days of exposure. These included all cancer sites combined, prostate cancer, lung cancer, colon cancer, all lymphohematopoietic cancers, and leukemia. The group of lymphohematopoietic cancers included the lymphomas, myeloma and the leukemias. Although there were only 4 cases of NHL in the lowest tertile of intensity-weighted exposure days, we included NHL in our analysis to compare results with the existing epidemiologic study of metribuzin exposure and NHL. Due to a lack of exposed cases in certain exposure groups, we were not able to examine the possible association between metribuzin use and glioma because there were only 4 cases of glioma in applicators who reported metribuzin use. To minimize the possibility of uncontrolled confounding from differences between pesticide applicators who used metribuzin and those who did not, we used the lowest exposed tertile of metribuzin users as the referent group to calculate rate ratios. However, we also report tests for linear trend using the nonexposed as the referent. Rate ratios were adjusted for age at enrollment ( $<40,40-49,50-59$, $60-69, \geq 70$ ), education ( $\leq$ high school, $>$ high school), smoking (never, $<12$ pack-years, $\geq 12$ pack-years), alcohol consumption during the last year (yes/no) family history of cancer (yes/ no), state of residence (Iowa/North Carolina), and exposure to all pesticides (tertiles of total lifetime exposure days). We used the Agricultural Health Study data release version P1REL0612.

\section{RESULTS}

Characteristics of applicators by their level of metribuzin exposure are shown in Table 1 . The "lowest exposed" category refers to those applicators in the lowest exposed tertile of lifetime exposure days. The "highest exposed" category refers to those applicators in the two highest tertiles of lifetime exposure days. There were some differences in the distribution of demographic characteristics among exposure groups. In general, applicators in Iowa were more likely to be exposed to metribuzin than applicators in North Carolina, and applicators in the highest exposure group were more likely to be commercial applicators than private applicators as compared to the low-exposed and non-exposed $(\mathrm{p}<0.0001)$. Additionally, applicators exposed to metribuzin tended to have higher exposures to all pesticides than the unexposed when asked to report their lifetime years and average days per year of use of pesticides overall $(\mathrm{p}<0.0001)$.

Of the 23,072 eligible applicators who provided detailed metribuzin use information on the take-home questionnaire, 8,504 (37 percent) reported using metribuzin. There were 554 incident cancer cases among those applicators who ever used metribuzin, compared to 1,118 cases of cancer in 14,568 applicators never used metribuzin. The rate ratio for all incident cancers in the exposed group compared to the unexposed group was 0.94 (95 percent confidence interval (CI): 0.84, 1.06) after adjustment for age, smoking, alcohol consumption, education, family history of cancer, state of residence, and exposure to all pesticides. The results from the ever/never analysis for the remainder of the specified cancer sites were close to the null with no statistically significant point estimates, although an elevated RR ( 95 percent CI) for leukemia of $1.40(0.77,2.56)$ was observed (data not shown).

Table 2 displays the associations between total lifetime days of metribuzin use and the incidence of selected cancers, adjusted for age, smoking, alcohol consumption, education, family history of cancer, state of residence, and use of all pesticides. An increased rate ratio was observed at the highest tertile of exposure using the low exposed referent group for all cancers combined and for several individual cancers, including leukemia, NHL, and all lymphohematopoietic cancers combined, although the point estimates and linear trend tests were not statistically significant. 
Table 3 shows the results of the analysis evaluating the adjusted associations between intensityweighted days of lifetime metribuzin exposure and the incidence of select cancers. Increased rate ratios were observed at the highest tertile of exposure for all cancers combined and for several individual cancers, including leukemia, NHL, and all lymphohematopoietic cancers combined. For all lymphohemopoietic cancers combined, a two-fold increased risk was observed in the highest exposure tertile; this association was largely driven by the positive association for leukemia, which contributed nearly half of the cases of lymphohematopoietic malignancies among exposed applicators. The test for linear trend for the lymphohematopoietic cancers using the low-exposed referent was statistically significant $(p=0.02)$, but only approached significance using the non-exposed referent $(\mathrm{p}=0.11)$. For leukemia, rate ratios increased with increasing exposure for the low-exposed referent group with greater than a twofold risk in the highest tertile exposure, although that point estimate was not significant. This monotonic increase was accompanied by a significant test for linear trend $(\mathrm{p}=0.04)$ including the non-exposed, but the linear trend test using the low-exposed only approached significance $(\mathrm{p}=0.08)$.

All analyses were repeated controlling for lifetime days of use (low, medium, high) to the five pesticides most highly correlated with metribuzin (cyanazine, chlorimuron-ethyl,

pendimethalin, butylate, and trifluralin) instead of exposure to all pesticides. These results did not differ substantively from those observed when controlling for exposure to all pesticides, and are not shown.

\section{DISCUSSION}

In this study, we did not observe an association between metribuzin use and overall cancer incidence. While we found a statistically significant association between leukemia and metribuzin in the highest tertile of intensity-weighted days which was accompanied by a significant linear trend test, these statistically significant findings were not consistent across exposure metrics and reference groups. We also found a significant trend test for the lymphohematopoietic cancers across tertiles of intensity-weighted exposure days using the low-exposed referent group, but the test only approached significance using the non-exposed referent. These differences may result from a smaller sample size when the low exposed group is used as the referent or may indicated residual confounding. Significant point estimates and tests for linear trend suggest that an association may exist between metribuzin and the increased risk of leukemia and all lymphohematopoietic cancers. In the absence of an a priori hypothesis or supporting epidemiologic, mutagenic, or genotoxic evidence, these positive results should be interpreted with caution. If these findings are confirmed, the relationship may suggest carcinogenesis through a non-genotoxic mechanism.

Our results indicate that there is not an overall increased risk of cancer in pesticide applicators enrolled in the Agricultural Health Study exposed to metribuzin. This is the first study to our knowledge that has examined the association between metribuzin exposure and the incidence of a variety of cancer sites in a human population.

The first epidemiologic study that examined the possible association between metribuzin and cancer did not find an association between metribuzin and NHL (13). This analysis was limited to an ever/never use categorization and a single cancer, and the retrospective design was not able to ensure that the exposures preceded the onset of disease. Because this was the only prior study exploring metribuzin exposure and cancer in humans, we included NHL in our analysis although we had a small number of cases. Our results for NHL using the non-exposed referent group were similar to those in the previous study. Point estimates for the medium and high exposure tertiles of both exposure metrics were elevated above the null for NHL using the lowexposed referent group; however, there was a significant inverse relationship in the lowest 
tertile of exposure compared to the non-exposed. Similar to the previous study which included 20 cases who reported ever being exposed to metribuzin (13), our results were based on small numbers of exposed cases $(\mathrm{n}=19)$, making interpretation more difficult.

The toxicologic evidence for metribuzin carcinogenicity is mixed. There are several negative tests for genotoxicity and mutagenicity (5,7-9); however, other in vitro genotoxicity and mutagenicity studies have yielded positive results $(10,11)$. It has been noted that herbicides in general do not directly interact with DNA, but may be able to induce tumors through other mechanisms which include promotion of spontaneous initiation, cytotoxicity, inhibition of apoptosis, oxidative stress, and formation of activated receptors (19). The potential for metribuzin to act as an epigenetic carcinogen combined with the presence of positive genotoxicity and mutagenicity tests in vitro and suggestive positive epidemiologic findings for leukemia warrants further exploration of possible carcinogenic effects of metribuzin exposure.

The Agricultural Health Study is the largest study of pesticide applicators exposed to metribuzin to date. A considerable strength of this study is the prospective cohort design. This design allowed the assessment of all pesticide and covariate exposure information prior to cancer diagnosis, which minimized recall bias in cases (15). The lifetime days metric provides an improvement over previous ever/never analysis of metribuzin use in humans. Use of this metric allowed us not only to quantify duration and frequency of exposure, but also allowed us to test for exposure-response effects. Additionally, we used a published exposure metric that was able to account for differences in intensity of exposure in addition to duration and frequency $(17,18)$. A comparison of the results of our analyses shows that the risk estimates were not meaningfully different when using the intensity-weighted metric as opposed to total lifetime days of exposure.

There are some limitations of this study. First, the investigation of certain cancers was hindered by small numbers of exposed cases. Additional follow-up in the AHS will provide more cases that will allow for more powerful analyses of many cancer sites. Second, pesticide applicators are frequently exposed to a variety of chemical, physical, and biological agents in agricultural work, which may potentially confound an exposure-disease relationship in regards to pesticides and cancer. There is the potential for confounding due to exposures that result from farmrelated activities, but investigators have determined that substantial bias in this cohort due to confounding from exposures resulting from performing other farming activities is not likely (20). Since $47 \%$ of the applicators completed the take-home questionnaire, there is the possibility for selection bias. However, participants who completed the take-home questionnaire were generally similar to the full cohort with the exception that responders were slightly older than non-responders, which may lead to higher farm-related exposures in responders (16). Also, since the full cohort provided information on ever use of metribuzin, we were able to compare the results of these analyses between the full cohort and the sub-group who completed the take-home questionnaire and found the results to be similar. Therefore, we believe selection bias is unlikely in this analysis. Exposure misclassification is another potential limitation of this study although this study provides more detailed information on usage of metribuzin than any other study of this chemical. The intensity-weighting algorithm took into account many factors that may affect the level of exposure, such as the use of personal protective equipment (PPE) and application practices. This information is not specific to metribuzin, but the intensity-weighting algorithm has been shown to be reasonably valid when comparing scores to the concentration of urine metabolite concentrations (18). In a prospective study such as this, it is almost certain that exposure misclassification is non-differential and would attenuate estimates of association. Finally, we included only men in this analysis so if use of metribuzin affects women's cancer risk differently, the results may not be generalizable. 
This study is the only prospective cohort study to examine the effects of metribuzin exposure and the risk of cancer in humans. Although the interpretation of the results from this study is limited at this time because of the paucity of cases for certain cancers, our results suggest that there is little or no association between metribuzin exposure and the incidence of all cancers combined. The results from this study suggest a potential association between metribuzin use and certain lymphohematopoietic malignancies in men; however, having not been observed previously caution should be used in interpretation. Additional follow-up in the Agricultural Health Study will provide more information on these risks as more cases arise.

\section{References}

1. Environmental Protection Agency. Metribuzin, R.E.D. Facts. Environmental Protection Agency, Office of Prevention, Pesticides, and Toxic Substances; Washington, DC: Feb. 1998 EPA 738R-97-006

2. Environmental Protection Agency. Health Effects Support Document for Metribuzin. Environmental Protection Agency; Washington, DC: 2003. EPA-733-R-04-001

3. International Programme on Chemical Safety. The WHO Recommended Classification of Pesticides by Hazard. World Health Organization; Geneva: 2002. http://www.who.int/ipcs/publications/en/pesticides_hazard.pdf)

4. Environmental Protection Agency. Integrated Risk Information System (IRIS): Metribuzin. Environmental Protection Agency; Washington, DC: Dec. 1993 http://www.epa.gov/iris/subst/0075.htm

5. Kaya B, Yanikoglu A, Creus A, Marcos R. Genotoxicity testing of five herbidices in the drorophila wing spot test. Mutation Research 2000;465:44-84.

6. Flores-Maya S, Gomez-Arroyo S, Calderon-Segura ME, et al. Promutagen activation of triazine herbicides metribuzin and ametryn through Vicia faba metabolism inducing sister chromatid exchanges in hyman lymphocytes in vitro and in $V$. faba root tip meristems. Toxicology in vitro 2005;19:243-251. [PubMed: 15649638]

7. Moriya M, Ohta T, Watanabe K, et al. Further mutagenicity studies on pesticides in bacterial reversion assay systems. Mutation Research 1983;116:185-216. [PubMed: 6339892]

8. Xu HH, Schurr KM. Genotoxicity of 22 pesticides in the microtitration SOS chromotest. Toxicology Assessment 1990;5:1-14.

9. Venkat JA, Shami S, Davis K, et al. Relative genotoxic activities of pesticides evaluated by a modified SOS microplate assay. Environmental Molecular Mutagenesis 1995;25:67-76.

10. Clements C, Ralph S, Petras M. Genotoxicity of select herbicides in Rana catesbeiana tadpoles using the alkaline single-cell gell DNA electrophoresis (comet) assay. Environmental and Molecular Mutagenesis 1997;29:277-288. [PubMed: 9142171]

11. Shah RG, Lagueux J, Kapur S, et al. Determination of genotoxicity of the metabolites of the pesticides Guthion, Sencor, Lorox, Reglone, Daconil, and Admire by ${ }^{32} \mathrm{P}$-postlabeling. Molecular and Cellular Biochemistry 1997;169:177-184. [PubMed: 9089646]

12. Bleeke MS, Smith MD, Casida JE. Metabolism and toxicity of metribuzin in mouse liver. Pesticide Biochemistry and Physiology 1985;23:123-130.

13. De Roos AJ, Zahm SH, Cantor KP, et al. Integrative assessment of multiple pesticides as factors for non-Hodgkin's lymphoma among men. Occupational and Environmental Medicine 2003;60:e11. [PubMed: 12937207]

14. Lee WJ, Colt JS, Heineman EF, McComb R, et al. Agricultural pesticide use and risk of glioma in Nebraska, United States. Occupational and Environmental Medicine 2005;62:786-792. [PubMed: 16234405]

15. Alavanja MC, Sandler DP, McMaster SB, et al. The Agricultural Health Study. Environmental Health Perspectives 1996;104:362-369. [PubMed: 8732939]

16. Tarone RE, Alavanja MCR, Zahm SH, et al. The Agricultural Health Study: Factors affecting completion and return of self-administered questionnaires in a large prospective cohort study of pesticide applicators. American Journal of Industrial Medicine 1997;31:233-242. [PubMed: 9028440] 
17. Dosemeci M, Alavanja MCR, Rowland AS, et al. A quantitative approach for estimating exposure to pesticides in the agricultural health study. Annals of Occupational Hygiene 2002;46:245-260. [PubMed: 12074034]

18. Coble J, Arbuckle T, Lee W, Alavanja M, Dosemeci M. The Validation of a Pesticide Exposure Algorithm Using Biological Monitoring Results. Journal of Occupational and Environmental Hygiene 2005;2:194-201. [PubMed: 15764542]

19. Rakitsky VN, Koblyakov VA, Turusov VS. Nongenotoxic (epigenetic) carcinogens: pesticides as an example. A critical review. Teratogenesis, Carcinogenesis, and Mutagenesis 2000;20:229-240.

20. Coble J, Hoppin JA, Engel L, et al. Prevalence of exposure to solvents, metals, grain dust, and other hazards among farmers in the Agricultural Health Study. J Expo Anal Environ Epidemiol 2002;12:418-26. [PubMed: 12415490] 


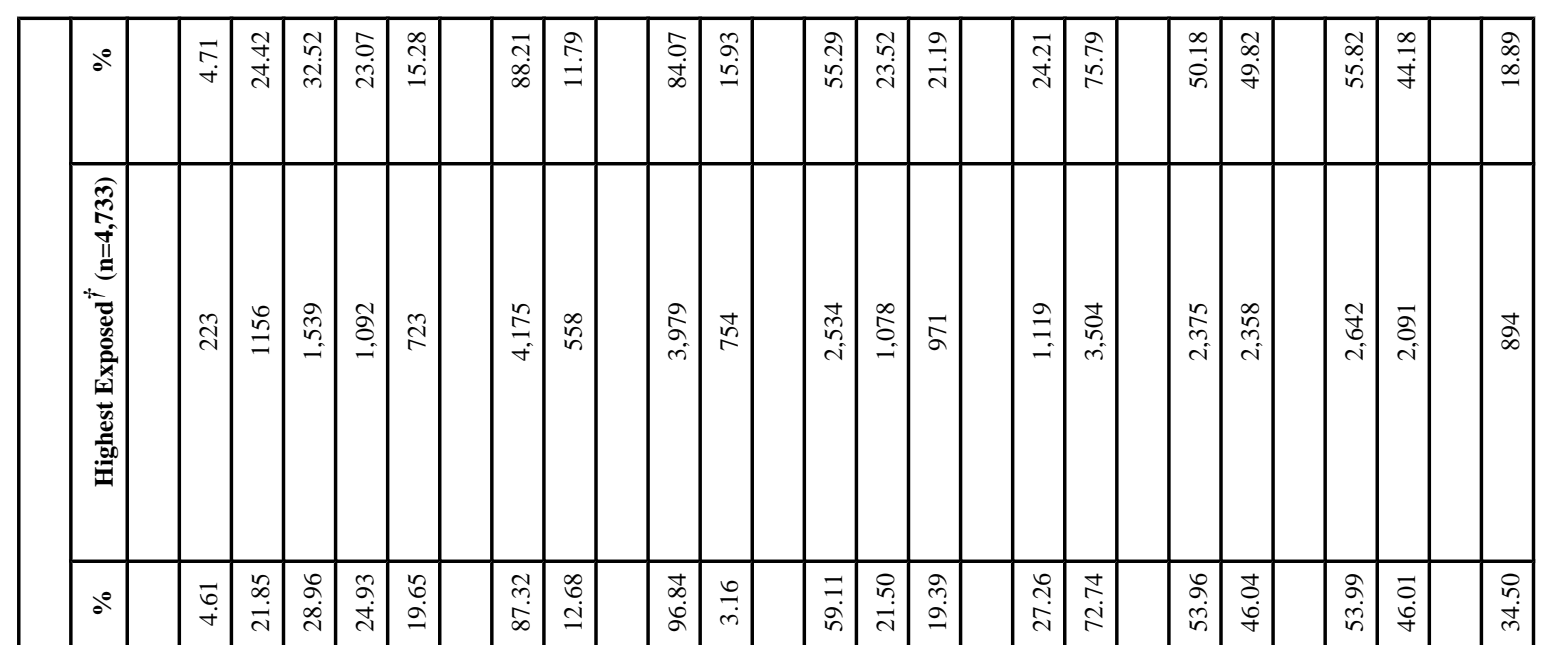

突

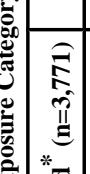

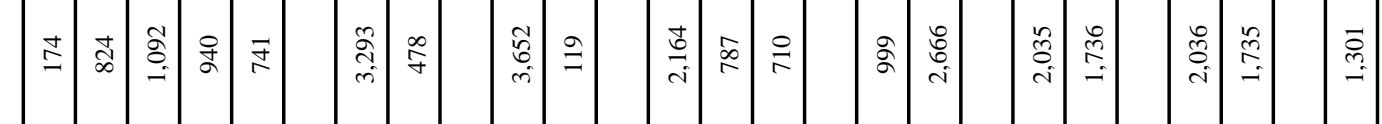

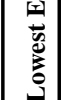

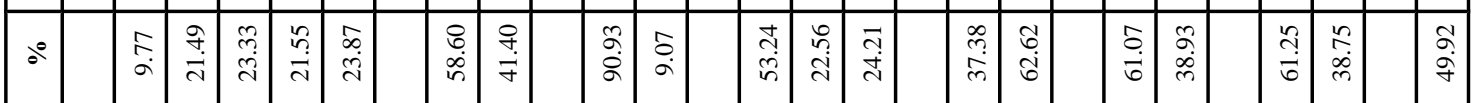




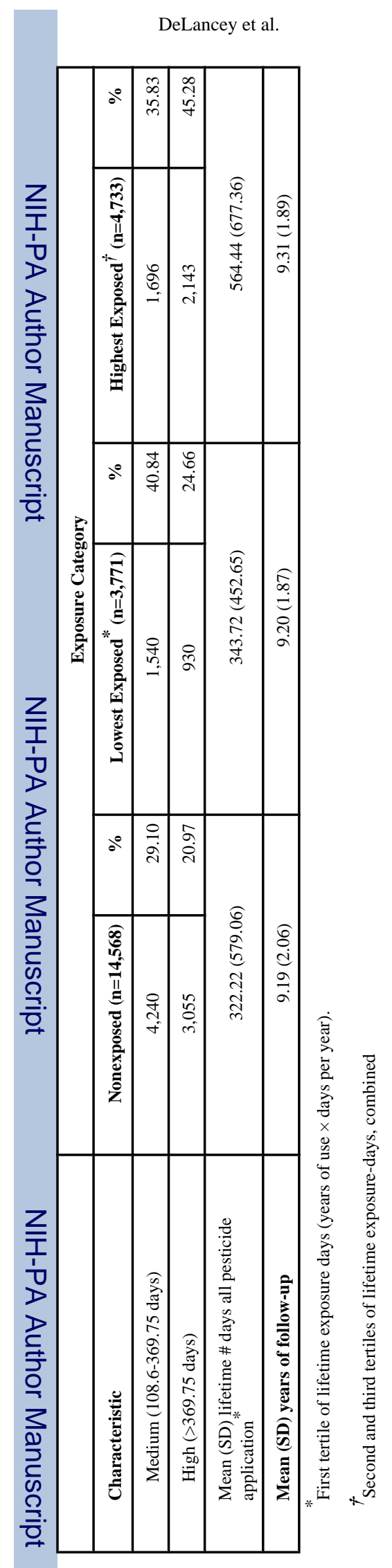

Page 10

Ann Epidemiol. Author manuscript; available in PMC 2009 September 1. 
Table 2

Rate Ratios* (RR) and $95 \%$ confidence intervals (CI) for selected cancer sites by total lifetime exposure days to metribuzin in male AHS members

\begin{tabular}{|c|c|c|c|c|}
\hline Cancer Site & Lifetime Exposure Days & Cases (n) & $\mathbf{R R}$ & $95 \% \mathrm{CI}$ \\
\hline \multirow[t]{5}{*}{ All Cancers } & 0 & 995 & & \\
\hline & $>0-8.75$ & 234 & 1.0 & \\
\hline & $8.76-24.5$ & 124 & 1.07 & $0.86,1.33$ \\
\hline & $>24.5$ & 158 & 1.13 & $0.92,1.39$ \\
\hline & & & \multicolumn{2}{|c|}{$\begin{array}{l}\mathrm{p}^{- \text {trend }}{ }^{\dagger}=0.21 \\
\text { p-trend }^{\dagger}=0.83\end{array}$} \\
\hline \multirow[t]{5}{*}{ Prostate } & 0 & 422 & & \\
\hline & $>0-8.75$ & 113 & 1.0 & \\
\hline & $8.76-24.5$ & 59 & 1.07 & $0.78,1.47$ \\
\hline & $>24.5$ & 63 & 0.99 & $0.72,1.35$ \\
\hline & & & \multicolumn{2}{|c|}{$\begin{array}{l}\text { p-trend }^{\dagger}=0.94 \\
\text { p-trend }\end{array}$} \\
\hline \multirow[t]{5}{*}{ Lung } & 0 & 109 & & \\
\hline & $>0-8.75$ & 16 & 1.0 & \\
\hline & $8.76-24.5$ & 8 & 1.08 & $0.46,2.52$ \\
\hline & $>24.5$ & 14 & 1.42 & $0.69,2.94$ \\
\hline & & & \multicolumn{2}{|c|}{$\begin{array}{l}\text { p-trend }^{\dagger}=0.41 \\
\text { p-trend }\end{array}$} \\
\hline \multirow[t]{5}{*}{ Colon } & 0 & 82 & & \\
\hline & $>0-8.75$ & 11 & 1.0 & \\
\hline & $8.76-24.5$ & 7 & 1.28 & $0.50,3.32$ \\
\hline & $>24.5$ & 14 & 2.07 & $0.94,4.60$ \\
\hline & & & \multicolumn{2}{|c|}{$\begin{array}{l}\text { p-trend }^{\dagger}=0.06 \\
\text { p-trend } \\
\text { t }^{\dagger}=0.98\end{array}$} \\
\hline \multirow[t]{5}{*}{ Lymphohematopoietic } & 0 & 83 & & \\
\hline & $>0-8.75$ & 19 & 1.0 & \\
\hline & $8.76-24.5$ & 12 & 1.21 & $0.59,2.51$ \\
\hline & $>24.5$ & 18 & 1.56 & $0.81,2.99$ \\
\hline & & & \multicolumn{2}{|c|}{$\begin{array}{l}\text { p-trend }^{\dagger}=0.27 \\
\text { p-trend } \\
{ }^{\dagger}=0.12\end{array}$} \\
\hline \multirow[t]{5}{*}{ Leukemia } & 0 & 27 & & \\
\hline & $>0-8.75$ & 9 & 1.0 & \\
\hline & $8.76-24.5$ & 6 & 1.38 & $0.49,3.88$ \\
\hline & $>24.5$ & 8 & 1.70 & $0.65,4.48$ \\
\hline & & & \multicolumn{2}{|c|}{$\begin{array}{l}\text { p-trend } d^{\dagger}=0.14 \\
\text { p-trend }\end{array}$} \\
\hline \multirow[t]{4}{*}{ NHL } & 0 & 39 & & \\
\hline & $>0-8.75$ & 5 & 1.0 & \\
\hline & $8.76-24.5$ & 6 & 2.31 & $0.62,8.63$ \\
\hline & $>24.5$ & 8 & 2.64 & $0.76,9.11$ \\
\hline
\end{tabular}




\begin{tabular}{|c|c|c|c|c|}
\hline Cancer Site & Lifetime Exposure Days & Cases (n) & $\mathbf{R R}$ & $95 \% \mathrm{CI}$ \\
\hline & & & \multicolumn{2}{|c|}{$\begin{array}{l}\mathrm{p} \text {-trend }{ }^{\dagger}=0.13 \\
\mathrm{p}^{+} \text {trend }^{+}=0.89\end{array}$} \\
\hline
\end{tabular}

* Each RR adjusted for age, smoking, alcohol consumption, education, family history of cancer, state of residence, and exposure to all pesticides ${ }^{\dagger}$ Test for trend, using the lowest exposed category as the referent

${ }^{\ddagger}$ Test for trend, using the non-exposed category as the referent 
Table 3

Rate ratios* (RR) and confidence intervals (CI) for selected cancer sites by intensity-weighted total lifetime exposure days to metribuzin among male AHS members

\begin{tabular}{|c|c|c|c|c|}
\hline Cancer Site & Intensity-Weighted Lifetime Exposure Days & Cases (n) & $\mathbf{R R}$ & $95 \% \mathrm{CI}$ \\
\hline \multirow[t]{5}{*}{ All Cancers } & 0 & 995 & & \\
\hline & $>0-58.3$ & 157 & 1.0 & \\
\hline & $>58.3-174.4$ & 183 & 1.04 & $0.84,1.29$ \\
\hline & $>174.4$ & 171 & 1.15 & $0.93,1.43$ \\
\hline & & & \multicolumn{2}{|c|}{$\begin{array}{l}\mathrm{p}_{\text {-trend }}^{\dagger}=0.21 \\
\mathrm{p}^{\dagger} \text {-trend }^{\ddagger}=0.69\end{array}$} \\
\hline \multirow[t]{5}{*}{ Prostate } & 0 & 422 & & \\
\hline & $>0-58.3$ & 71 & 1.0 & \\
\hline & $>58.3-174.4$ & 87 & 1.12 & $0.82,1.53$ \\
\hline & $>174.4$ & 74 & 1.17 & $0.84,1.63$ \\
\hline & & & \multicolumn{2}{|c|}{ 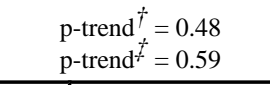 } \\
\hline \multirow[t]{5}{*}{ Lung } & 0 & 109 & & \\
\hline & $>0-58.3$ & 14 & 1.0 & \\
\hline & $>58.3-174.4$ & 10 & 0.65 & $0.29,1.47$ \\
\hline & $>174.4$ & 13 & 0.92 & $0.43,1.97$ \\
\hline & & & \multicolumn{2}{|c|}{$\begin{array}{l}\mathrm{p} \text {-trend }^{\dagger}=0.99 \\
\mathrm{p} \text {-trend }\end{array}$} \\
\hline \multirow[t]{5}{*}{ Colon } & 0 & 82 & & \\
\hline & $>0-58.3$ & 8 & 1.0 & \\
\hline & $>58.3-174.4$ & 11 & 1.25 & $0.50,3.10$ \\
\hline & $>174.4$ & 13 & 1.69 & $0.69,4.10$ \\
\hline & & & \multicolumn{2}{|c|}{$\begin{array}{l}\text { p-trend } \\
\text { p-trend }\end{array}$} \\
\hline \multirow[t]{5}{*}{ Lymphohematopoietic } & 0 & 83 & & \\
\hline & $>0-58.3$ & 11 & 1.0 & \\
\hline & $>58.3-174.4$ & 16 & 1.26 & $0.58,2.72$ \\
\hline & $>174.4$ & 22 & 2.07 & $0.99,4.29$ \\
\hline & & & \multicolumn{2}{|c|}{$\begin{array}{l}\mathrm{p} \text {-trend } \mathrm{d}^{\dagger}=0.02 \\
\mathrm{p} \text {-trend }\end{array}$} \\
\hline \multirow[t]{5}{*}{ Leukemia } & 0 & 27 & & \\
\hline & $>0-58.3$ & 5 & 1.0 & \\
\hline & $>58.3-174.4$ & 8 & 1.45 & $0.47,4.45$ \\
\hline & $>174.4$ & 10 & 2.42 & $0.82,7.19$ \\
\hline & & & \multicolumn{2}{|c|}{$\begin{array}{l}\mathrm{p} \text {-trend }{ }^{\dagger}=0.08 \\
\text { p-trend }\end{array}$} \\
\hline \multirow[t]{4}{*}{ NHL } & $>0-58.3$ & 39 & & \\
\hline & $>58.3-174.4$ & 4 & 1.0 & \\
\hline & $>174.4$ & 6 & 1.39 & $0.33,5.84$ \\
\hline & $>0-58.3$ & 9 & 2.52 & $0.66,9.59$ \\
\hline
\end{tabular}




\begin{tabular}{|l|c|r|r|r|}
\hline Cancer Site & Intensity-Weighted Lifetime Exposure Days & Cases (n) & RR & 95\% CI \\
\hline & & & \multicolumn{2}{|c|}{$\begin{array}{c}\mathrm{p}^{- \text {trend }^{\dagger}}=0.13 \\
\text { p-trend }\end{array}=0.72$} \\
\hline
\end{tabular}

* Each RR adjusted for age, smoking, alcohol consumption, education, family history of cancer, state of residence, and exposure to all pesticides ${ }^{\dagger}$ Test for trend, using the lowest exposed category as the referent

${ }^{*}$ Test for trend, using the non-exposed category as the referent 